\title{
Avaliação da percepção dos alunos de graduação sobre a utilização de instrumentos manuais de NiTi
}

Evaluation of undergraduate students perception of the use of NiTi hand instruments

Evaluación de la percepción de los estudiantes universitarios sobre el uso de instrumentos manuales NiTi

Alexandre Moreira FERNANDES ${ }^{\mathbf{1}}$

Ana Cláudia Souza SODRÉ ${ }^{2}$

Isadora Santana MOREIRA ${ }^{2}$

Lara Duarte MOURA ${ }^{2}$

${ }^{1}$ Professor de Endodontia no Centro Universitário Newton Paiva, 30341-262 Belo Horizonte, MG, Brasil

${ }^{2}$ Curso de Graduação em Odontologia, Centro Universitário Newton Paiva, 30341-262 Belo Horizonte, MG, Brasil

\section{Resumo}

As limas de ligas com NiTi vieram para modernizar e facilitar a vida dos estudantes ao realizarem tratamentos endodônticos, apresentando grandes vantagens em relação a qualidade do aprendizado dos mesmos. No entanto, muitas instituições não acompanham a evolução da endodontia, que como comprovada pode não ser a melhor opção. O projeto de pesquisa foi submetido ao Comitê de Ética e Pesquisa Humana, por meio da Plataforma Brasil. Esse trabalho foi realizado no Centro Universitário Newton Paiva em Belo Horizonte MG, com o objetivo de avaliar a percepção de 14 estudantes de odontologia, ao realizarem tratamentos endodônticos executados com instrumentos manuais de NiTi em comparação com os tradicionais de aço inoxidável, por meio de um questionário estruturado com sete perguntas observando os seguintes tópicos: percepções sobre o aprendizado, percepções sobre as características das técnicas, experiência na prática endodôntica e avaliação geral das técnicas pelos alunos. Os resultados mostraram que os estudantes percebem a experiência clínica como sendo o aspecto mais importante da sua educação odontológica, independente do seu ano na graduação. Dado que, a experiência é alcançada pela maior exposição aos pacientes, sendo adquirida por instrumentos que proporcionam maior agilidade, facilidade, simplicidade e entendimento dos tratamentos. Em síntese, as limas manuais de NiTi Pro Design- M (PDM) devem ser instituídas na graduação odontológica.

Descritores: Endodontia; Preparo de Canal Radicular; Educação.

\section{Abstract}

The files of alloys with NiTi came to modernize and facilitate the life of the students when performing endodontic treatments, presenting great advantages in relation to the quality of their learning. However, many institutions do not follow the evolution of endodontics, which as proven may not be the best option. The research Project was submitted to the "Ethics and Human Research Committee", through the "Plataforma Brasil". This Work was perfomed at Newton Paiva University Center in Belo Horizonte MG, with the objective of evaluating the perception of 5 dental students when perfoming endodontic treatments perfomed with manual instruments of NiTi compared to the traditional stainless steel, through a structured questionnaire with seven questions observing the following topics: perceptions about learning, perceptions about the characteristics of techniques, experience in endodontic practice and general evaluation of techniques by students. The results will show that students are aware of clinical experience as being or most important aspect of their dental education and experience and reach the highest exposure to patients, being acquired by instruments that provide greater agillity, ease, simplicity and understanding of two treatments. In other words, the NiTi Pro Design M (PDM) manual files must be instituted without a graduate dental curriculum. In summary, the manual files of NiTi Pro Design M (PDM) should be instituted in dental graduation.

Descriptors: Endodontics; Root Canal Preparation; Education.

\section{Resumen}

Las limas de liga con NiTi vinieron para modernizar y facilitar la vida de los estudiantes al realizar tratamientos endodónticos, presentando grandes ventajas en relación a la calidad del aprendizaje de los mismos. No obstante, muchas intituiciones no acompañan la evolución de la endodoncia, que tiene comprobado que no puede ser la mejor opción. El proyecto de investigación fue sometido al Comité de Ética de la Investigación en humanos, a través de la " Plataforma Brasil ". Este trabajo fue realizado em el Centro Universitário Newton Paiva em Belo Horizonte MG, com el objetivo de evaluar la percepción de 14 estudiantes de odontologia, al realizarse tratamientos endodónticos ejecutado com instrumentos de mano em NiTi comparado con los tradicionales en acero inoxidable, a través de un cuestionario estructurado con siete preguntas, observamos los siguientes temas: percepción del aprendizaje, percepción acerca de las características de las técnicas, experiencia en práctica de endodoncia y eyaluación general de técnicas por los estudiantes. Los resultados mostraron que los estudiantes percebem la experiência clínica como el aspecto más importante de la odontología, independiente te de tu año de graduacíon. Dado que, la experiencia se logra com la mayor exposición a los pacientes siendo adquirida por instrumentos que brindan mayor agilidad, facilidad, simplicidad y comprensión de los tratamientos. Sin embargo, la lima de mano em NiTi "Pro Desing- M (PDM) " debe ser instituído en la graduación de odontologia.

Descriptores: Endodoncia; Preparación del Conducto Radicular; Educación.

\section{INTRODUÇÃO}

Durante o ensino de endodontia nas faculdades de odontologia, a maioria das instituições brasileiras utilizam instrumentos de aço inoxidável. No entanto, os principais erros de procedimento durante a instrumentação como transportes e degraus se devem à rigidez dessa liga metálica, principalmente em condutos curvos $^{1}$. As dificuldades inerentes à instrumentação com esse tipo de instrumento contribuem para percepções negativas dos estudantes sobre a endodontia. Segundo os alunos, é necessário um grande período de treinamento, sem o qual a falta de experiência clínica torna a instrumentação endodôntica um procedimento difícil e demorado ${ }^{2}$. Desde sua descoberta, até a aplicação para a confecção de instrumentos endodônticos, a liga de NiTi têm apresentado inúmeras vantagens em relação ao aço inoxidável. Sua flexibilidade proporcionada pelo menor módulo de elasticidade, por exemplo, diminui consideravelmente as principais falhas relacionadas à formatação ${ }^{3,4}$ 
gerando aplicação clínica com melhores resultados, mesmo entre operadores inexperientes ${ }^{5}$.

A implementação de instrumentos de $\mathrm{NiTi}$ na graduação ocorre principalmente através de sistemas mecanizados (rotatórios e reciprocantes) e estudos mostram resultados promissores $^{5-7}$. Contudo, o rompimento desses instrumentos utilizados mecanicamente, mesmo obedecidas todas as normas de segurança e realizados treinamentos necessários nos operadores ainda se mostra uma realidade a ser enfrentada ${ }^{8}$.

Visando aproveitar as vantagens da liga de NiTi na endodontia e procurando diminuir o risco de fratura de instrumentos, possivelmente aumentado quando os estudantes se utilizam de sistemas mecanizados, foram desenvolvidos os instrumentos ProDesign M (Easy Equipamentos Odontológicos - Belo Horizonte MG Brasil). O sistema consiste em limas de NiTi do tipo CM ("Controlled Memory") adaptadas para utilização manual. Para formatação de dentes multirradiculares a técnica propõe a utilização de três instrumentos $(25.01,15.05$ e 25.06) de secções transversais quadrangulares e duplohelicoidais.

Até o presente momento, não se observa na literatura nenhum trabalho avaliando a percepção de alunos de graduação a respeito desses novos instrumentos, procurando detalhar possíveis vantagens ou dificuldades em relação aos instrumentos tradicionalmente utilizados (aço inox). Assim, o presente trabalho possui o objetivo de analisar as percepções de estudantes de graduação em relação à utilização dos instrumentos ProDesign $M$ em pacientes, comparando-as aos métodos tradicionais de formatação através de instrumentos de aço inox.

MATERIAL E MÉTODO

Esse estudo contou com a participação de 14 estudantes de graduação regularmente matriculados no $7^{\circ}$ e $9^{\circ}$ semestres do curso de odontologia do Centro Universitário Newton Paiva, Belo Horizonte, estado de Minas Gerais, Brasil, os alunos foram livremente convidados, mas desde que já tenham cursado a prática clínica em endodontia, realizado algum tratamento endodôntico com os instrumentos preconizados pela instituição, afim de comparação, estejam dispostos a adquirirem as limas manuais de NiTi e que tenham interesse em participar da pesquisa.

$\mathrm{O}$ projeto de pesquisa foi submetido a aprovação pelo Comitê de Ética e Pesquisa Humana por meio da Plataforma Brasil
(Protocolo $\mathrm{n}^{\circ}$ 68475617.0.0000.5097), tanto os alunos quanto os pacientes que manifestaram interesse, assinaram um Termo de Consentimento Livre e Esclarecido (TCLE). Os dados obtidos foram utilizados somente para fins de pesquisa, sendo suprimidas quaisquer informações que possam permitir a identificação dos participantes, não estando estes expostos a qualquer risco de exposição ou danos. De acordo com os preceitos éticos, o anonimato dos participantes irá ser preservado durante todo o estudo.

O curso de graduação do Centro Universitário Newton Paiva possui um currículo que consiste em 9 semestre para os turnos da manhã e tarde e 10 semestres para o turno da noite. A disciplina Endodontia é ministrada durante o quinto, sexto, sétimo e nono períodos, da seguinte forma. No quinto período há aula teórica e pré-clínico no laboratório (com média de $20 \mathrm{~h}$ teoria e $20 \mathrm{~h}$ pré-clínico), no sexto período há aula teórica e clínica com tratamentos em dentes unirradiculares, sendo 40 h teoria e 180 h prática, no sétimo há clínica para tratamento em dentes multirradiculares, são 180 h de prática clínica, onde o aluno pode realizar somente procedimentos de endodontia ou de outras disciplinas como prótese, cirurgia e dentística, já no nono período são 80 h de prática clínica na faculdade e 220 h de estágio, onde 0 aluno pode realizar qualquer procedimento que tenha demanda.

A matriz curricular 2014-1 do curso de Odontologia utilizava a técnica híbrida (coroaápice/ ápice coroa) somente com limas manuais de aço-inoxidável para instrumentação dos canais radiculares, porém houve uma reformulação na grade curricular em 2017-1, inserindo também as limas manuais de NiTi denominados ProDesign $\mathrm{M}$, produzidas pela EASY Equipamentos Odontológicos (Belo Horizonte, Minas Gerais, Brasil).

A fim de avaliar a percepção dos alunos de graduação sobre estes instrumentos, foi dado início ao estudo, em que, os alunos foram orientados e treinados em como utilizar os instrumentos (Sistema ProDesing M) em forma de pré-clínico pelo professor orientador. A técnica preconizada para 0 tratamento endodôntico pela equipe de Endodontia da Newton Paiva é a técnica híbrida (coroa-ápice / ápice coroa) e utiliza instrumentos manuais de aço-inoxidável e a técnica endodôntica sugerida neste estudo utiliza instrumentos de $\mathrm{NiTi}$ denominados ProDesign M. É importante destacar que ambas as técnicas foram realizadas nos pacientes em dentes permanentes, uni ou multirradiculares. Os 
tratamentos foram selecionados minuciosamente por radiografias analógicas para em um segundo momento serem comparadas.

Após a realização do teste com os instrumentos (ProDesign M), a coleta de dados foi iniciada, por meio da elaboração de uma ficha informativa, onde constam informações acerca da natureza do trabalho e seus objetivos. Foi avaliada a percepção dos alunos, em relação aos dois tipos de instrumento, através de um questionário estruturado aplicado para os alunos, com sete perguntas observando os seguintes tópicos: percepções sobre 0 aprendizado, percepções sobre as características das técnicas, experiência na prática endodôntica e avaliação geral das técnicas pelos alunos.

Em seguida, os dados foram tabulados, analisados e codificados em sete variáveis pelo teste de Wilcoxon. Além disso, foi acrescentado radiografias analógicas, disponibilizadas pelo próprio Centro Universitário, contribuindo na avaliação, acompanhamento e conclusão dos casos clínicos.

\section{RESULTADOS}

Amostra de 14 alunos dos últimos períodos de Odontologia (10 do $7^{\circ}$ Período, 3 do

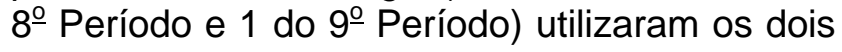
tipos de instrumentos: Aço Inox e NiTi (Pro Design M).

O objetivo da pesquisa foi comparar a percepção dos alunos em relação a cada tipo de instrumento. Com esta finalidade foi elaborado um questionário e foram coletadas informações relativas a diversos tópicos: qualidade do aprendizado, grau de ansiedade, dificuldade encontrada, tempo gasto, qualidade do tratamento, característica da técnica e quantidade de tratamento realizado.

A Tabela 1 resume as observações coletadas e o resultado do teste estatístico comparativo entre os dois tipos de limas.

Os gráficos 1 a 7 (Figuras 1 a 7 ) ilustram de forma visual, os dados da Tabela 1.

Reunindo os dados obtidos de todas as fases da Pesquisa, e fazendo o Teste comparativo para amostras emparelhadas, Teste de Wilcoxon, o resultado obtido se encontra na Tabela-1: GERAL p-valor $=0.001$ bastante significativo mostrando que a Lima da liga NiTi obteve resultados superiores às de Aço inox.

Considerando cada questão avaliada, os testes estatísticos se mostraram significativamente diferentes de zero quando comparamos os dois tipos de instrumentos sendo a de NiTi com desempenho superior à de Aço, exceto no caso da Quantidade de tratamentos, mesmo assim este resultado é significativo com $\alpha=10 \%$. Em todos os outros casos usamos $\alpha=5 \%$.

Tabela 1. Respostas ao questionário
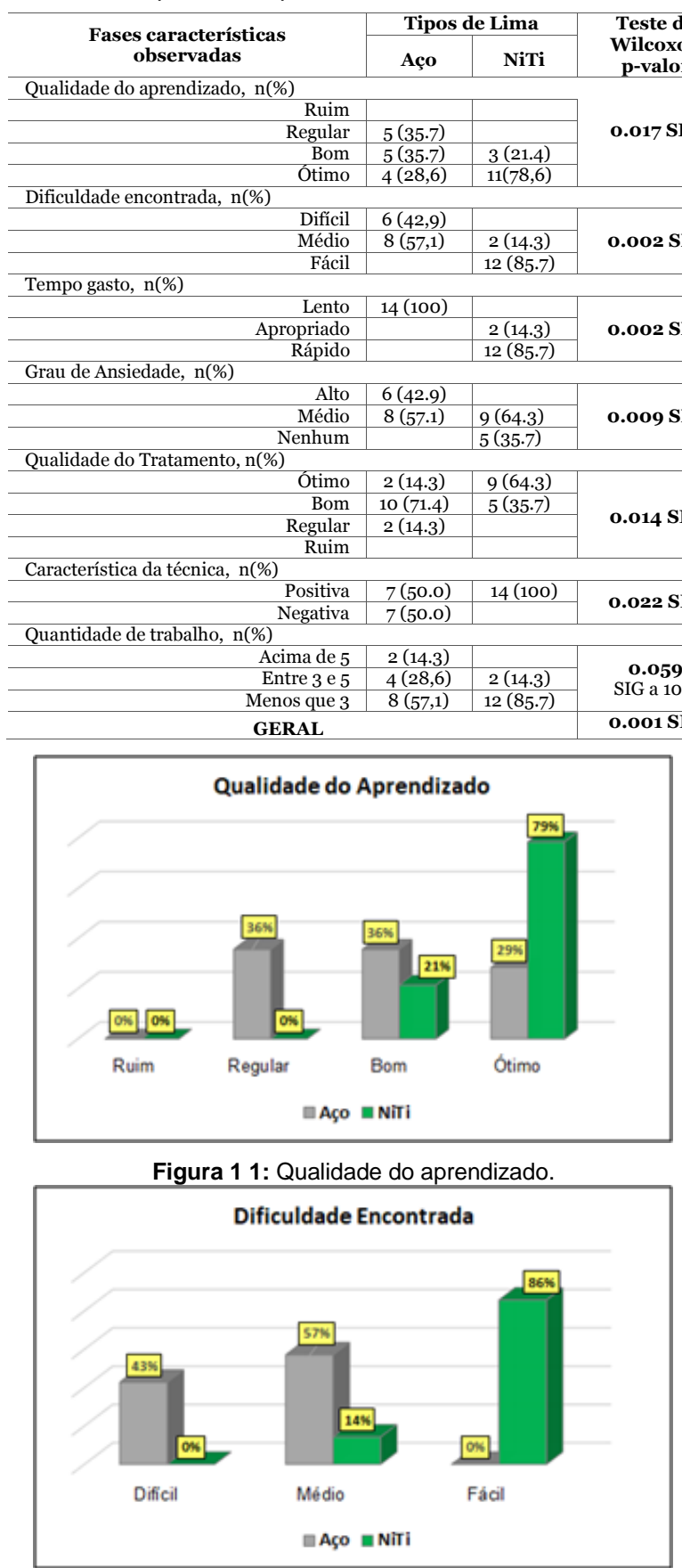

Figura 2: Dificuldade encontrada.

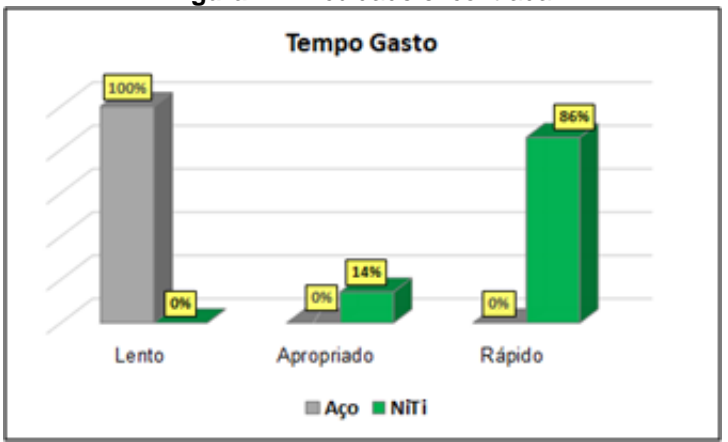

Figura 3: Tempo gasto. 


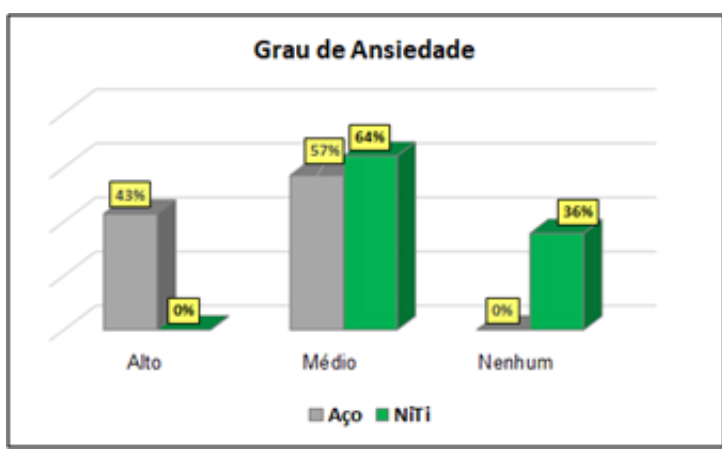

Figura 4: Grau de ansiedade.

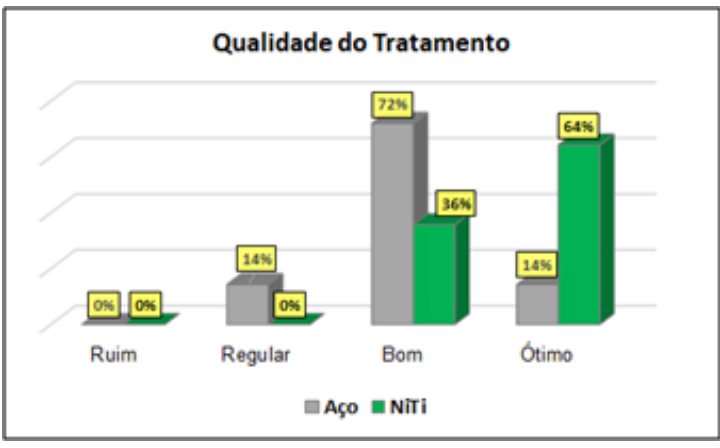

Figura 5: Qualidade do tratamento.

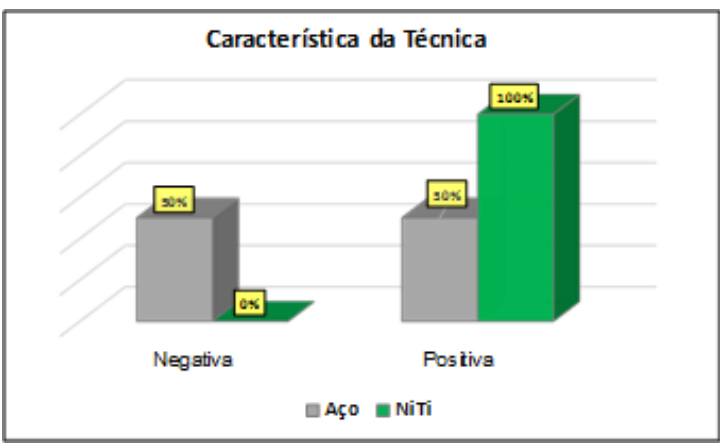

Figura 6: Característica da técnica.

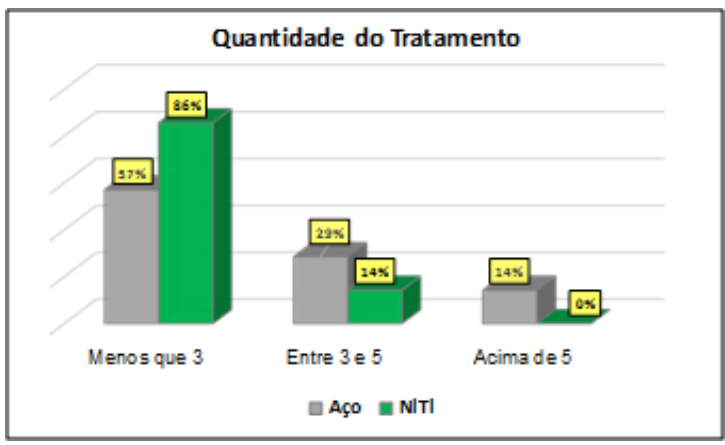

Figura 7: Quantidade do Tratamento.

DISCUSSÃO

Um questionário foi utilizado para este estudo em modelo de teste, e foi aplicado presencialmente, em clínica, após a última etapa do tratamento endodôntico dos alunos, a obturação, para evitar abstrações, desleixos e garantir precisão do teste. A decisão de fazer o questionário foi devido a esta ser a melhor forma de se avaliar percepções de certa amostra e, aos resultados positivos encontrados em diversos estudos ${ }^{2,7,10,11}$. O questionário foi elaborado para ser rápido e fácil de concluir e teve uma taxa de resposta de $100 \%$, o que foi adequado para fornecer dados significativos. Entretanto, variabilidades podem estar presentes em outros estudos ${ }^{7}$, obteve taxa de resposta de $91,3 \%$ por ser um modelo testereteste e ter sido entregue nas provas finais dos alunos. Ao passo que, essa variabilidade pode ocorrer devido a forma que o questionário é apresentado, por exemplo em forma de correio eletrônico, como feito por ${ }^{11}$ que obteve taxa de $51 \%$ a $76 \%$ devido a mudança de opinião dos alunos durante o tempo de pesquisa.

De maneira quantitativa este estudo observou que a falta de autoconfiança dos estudantes, os induzem a não se sentirem preparados para realizarem os procedimentos, devido a diversos fatores, tais como: falta de motivação uma vez que as técnicas preconizadas são de alto custo, sistemática complexa gerando assim dificuldade dos estudantes no aprendizado, longa duração (pela inexperiência nos procedimentos). Tudo isso resulta em baixa exposição aos pacientes e menor experiência no âmbito clínico, além de estresse e dificuldade dos alunos com a endodontia.

Em relação ao "nível de dificuldade encontrado no tratamento", o estudo obteve porcentagem de $57,1 \%$ para os de aço e $14,3 \%$ para os de NiTi com grau de dificuldade médio, 42,9\% para o de aço e 0\% para o de NiTi para os alunos que consideraram difícil e $0 \%$ para os de aço e $85,7 \%$ para os de $\mathrm{NiTi}$ com grau de dificuldade fácil. Isso pode ser atribuído à diferença entre as ligas metálicas e a cinemática de cada instrumento. ${ }^{9}$ A liga de aço inoxidável não possui flexibilidade, acarretando em um ao alto índice de fratura, sua sistemática complexa leva a uma longa duração nos procedimentos e dificuldade de entendimento dos alunos ${ }^{5}$. Além disso, os instrumentos de aço inoxidável têm uma capacidade de centralização difícil, com uma tendência de transporte em direção à direção mesial do canal. Uma forma de solução para esses problemas seria a introdução de instrumentos com maior simplicidade, técnicas mais rápidas, mas com maior eficiência proporcionando aos alunos maior confiança nos procedimentos e maior exposição a pacientes ${ }^{4}$. A universidade Newton Paiva já está bem encaminhada para mudanças nos protocolos visando melhorias para os alunos, através da substituição das ligas de aço inox por NiTi.

O tempo reduzido necessário para a realização de tratamentos endodônticos foi observado no presente estudo. De acordo com os alunos, "o tempo gasto" na realização do tratamento endodôntico com os instrumentos manuais foi $100 \%$ lento para os de aço inox, 
$14,3 \%$ apropriado para os de $\mathrm{NiTi}$ e $85,7 \%$ foi considerado rápido para os $\mathrm{NiTi}$. No entanto, esse resultado não foi uma variável calculável, pois o tempo não foi cronometrado e sim avaliado a percepção dos alunos em relação ao tempo. Esse fato pode ser explicado pela sistemática complexa, com o maior número de etapas e instrumentos utilizados com a técnica de limas de aço inox, além de outros fatores que influenciam o tempo gasto durante o tratamento endodôntico, no qual segundo os estudantes, são o não atendimento dos pacientes, a falta de experiência clínica e as dificuldades com a exposição das radiografias ${ }^{2,7,11}$.

Outro problema, reconhecido pelos estudantes, foi a "quantidade de tratamentos realizados". O resultado do estudo mostrou que $57,1 \%$ de estudantes relataram ter realizado menos que 3 procedimentos utilizando instrumentos de aço e $85,7 \%$ utilizando os de $\mathrm{NiTi}, 28,6 \%$ realizaram entre 3 e 5 tratamentos utilizando o aço inoxidável e $14,3 \% \mathrm{NiTi}$. Já os alunos que realizaram acima de 5 tratamentos endodônticos, foram 14,3\% utilizando a liga de aço inox. Isso pode ser explicado pela baixa experiência dos alunos nos tratamentos em geral, pela técnica empregada em que a característica da liga usada influencia muito e o tempo curto de clínica. Com as limas de liga de $\mathrm{NiTi}$, os alunos compraram, aprenderam e começaram a utilizar no próprio semestre em que cursavam, além de terem concluído outros procedimentos, influenciando assim o número de tratamentos que eles poderiam ter finalizado $^{2,7}$. Os tratamentos endodônticos com menos tempo e menos sessões, aumentam o número de procedimentos que os alunos são capazes de realizar ${ }^{6}$.

Uma das variáveis mais discutidas é sobre a "qualidade do aprendizado", mostrando que $21,4 \%$ consideraram ótimo utilizando o aço, em comparação com $78,6 \%$ de $\mathrm{NiTi}$, já $35,7 \%$ consideraram os de aço bom e $21,7 \%$ os de $\mathrm{NiTi}$. Cerca de $35,7 \%$ consideraram a qualidade de aprendizado com o aço regular. Já em relação a "qualidade do tratamento" notou-se que a porcentagem dos alunos que consideraram ótimo foi de $14,3 \%$ de aço e $64,3 \%$ de $\mathrm{NiTi}$, já os que consideram bom utilizando o aço inox foi de $71,4 \%$ e $35,7 \%$ o $\mathrm{NiTi}$ e 14,3\% relataram a qualidade ter sido regular usando o aço inoxidável. Em relação a "característica da técnica" somente os estudantes que utilizaram os instrumentos manuais de aço inoxidável atribuíram características negativas a técnica empregada sendo esses $50 \%$. Em contrapartida, todos os alunos que utilizaram os manuais de $\mathrm{NiTi}$ consideraram positivo. Isso se deve ao fato das limas de ligas manuais de $\mathrm{NiTi}$ apresentarem uma técnica mais simples, com menor número de etapas, sendo assim menos complexa fazendo com que os estudantes aprendam de forma mais fácil e rápida. Os resultados mostraram que o tempo de preparação da raiz dos canais foi menor utilizando os instrumentos manuais de NiTi sistema Pro- Design $\mathrm{M}$ em comparação com os de aço inoxidável, devido o número de instrumentos para realizar o tratamento, menor sequência de passo a passo e melhor capacidade de centralização por sua flexibilidade. Isso pode ser atribuído a diferença entre as ligas metálicas e a cinemática de cada instrumento ${ }^{9}$. Apesar das dificuldades encontradas durante o tratamento endodôntico, os estudantes consideraram seus tratamentos bons. A qualidade atribuída aos procedimentos realizados pelos graduandos relacionou-se principalmente a análise radiográfica e erros de procedimentos.

O resultado do estudo mostrou que o "grau de ansiedade ou preocupação durante o tratamento" apresentou $57,1 \%$ para o aço inoxidável e 64,3\% com grau de ansiedade nível médio, $42,9 \%$ para os de aço e $0 \%$ para o de $\mathrm{NiTi}$ no nível alto. Já os que não relataram ansiedade foi $0 \%$ para os de aço e $35,7 \%$ para os de NiTi. A maioria dos estudantes relataram sentir o mesmo grau de ansiedade com as duas técnicas e esse valor pode ser devido a diversos motivos como: má interpretação dos alunos ou da não compreensão das perguntas feitas, pelo duplo sentido que a pergunta pode ter gerado. Os alunos podem ter entendido que o grau de ansiedade foi referente a "ansiedade em realizar algo novo" e não pela técnica propriamente dita ser complexa, por exemplo. Esse valor também pode ser explicado pela bagagem de experiências negativas que os estudantes carregam com eles e esse problema não é restrito à nossa escola, podendo estar relacionado a experiências com as técnicas tradicionais, com aço inox, preconizadas por muitas instituições ${ }^{11}$. O problema da ansiedade está na capacidade de poder influenciar no potencial dos alunos. A falta de confiança percebida tendia a estar relacionado à ansiedade e não à falta de competência. Portanto, enquanto vários alunos relatam sentirse mal preparado para as avaliações, é provável que isso seja influenciado pela ansiedade ${ }^{10}$.

No quinto período há aula teórica e préclínico no laboratório em tempo curto (com média de 20 horas teóricas e 20 horas préclínico), no sexto os alunos já colocam o treinamento do laboratório em prática clínica em 
dentes unirradiculares, no sétimo e nono fazem atendimento aos pacientes e em dentes multirradiculares. No entanto, a clínica é multidisciplinar sendo da escolha do aluno em qual área atuar (endodontia, dentística, prótese e cirurgia). Diante disso, o tempo limitado para treinamento clínico e pré-clínico em Endodontia pode resultar em baixa autoconfiança durante a prática clínica e, as autoconfianças dos alunos podem ser aumentadas pela maior exposição a procedimentos clínicos e a pacientes. Esses fatos podem explicar a insatisfaço dos alunos observadas nas variáveis.

Como qualquer estudo qualitativo, nossa análise de dados está sujeita a víeis de interpretação como: o pequeno número de pessoas avaliadas, curto tempo para pesquisa, possibilidade de inverdades dos contribuintes, a condição dos alunos terem que adquirir comercialmente as limas que não foram financiadas pelos organizadores do estudo ou pela faculdade, a forma que o questionário foi aplicado não contendo um reteste, o tempo não ter sido uma variável calculável e o fato dos participantes terem que executar outros procedimento durante cada semestre na clínica, por exemplo, prótese, dentística e cirurgia.

As evidências suportam uma mudança no ensino para uma que os alunos possam dominar mais facilmente, ter menos risco de erros processuais, ser mais rápida e ter um resultado clínico aprimorado. Não foram produzidas evidências para fundamentar a opinião de que os alunos devem primeiro se tornar competentes com os arquivos manuais antes de usar os arquivos rotativos ${ }^{6}$. Portanto, aqueles que usaram a técnica manual em aço inox no estudo tinham maior familiaridade do que aqueles que usavam as técnicas manuais de NiTi. No entanto, maior familiaridade não se traduz em maior competência. Isso está de acordo com os resultados encontrados obtidos em outros estudos por ${ }^{6}$.

CONCLUSÃO

Em suma, os estudantes percebem a experiência clínica como sendo o aspecto mais importante da sua educação odontológica, independente do seu ano na graduação. Dado que, a experiência é alcançada pela maior exposição aos pacientes e é adquirida por instrumentos que proporcionam maior agilidade, facilidade, simplicidade e entendimento dos tratamentos, as limas manuais de $\mathrm{NiTi}$ Pro Design (PDM) devem ser instituídas no currículo odontológico.

\section{REFERÊNCIAS}

1. Bahia MG, Melo MC, Buono VT. Influence of simulated clinical use on the torsional behavior of nickel-titanium rotary endodontic instruments. Oral Surg Oral Med Oral Pathol Oral Radiol Endod. 2006;101(5):675-80.

2. Seijo MO, Ferreira EF, Ribeiro Sobrinho AP, Paiva SM, Martins RC. Learning experience in endodontics: Brazilian students' perceptions. J Dent Educ. 2013;77(5):648-55.

3. Walia HM, Brantley WA, Gerstein H. An initial investigation of the bending and torsional properties of Nitinol root canal files. J Endod. 1988;14(7):346-51.

4. Zhou HM, Shen Y, Zheng W, Li L, Zheng YF, Haapasalo M. Mechanical properties of controlled memory and superelastic nickeltitanium wires used in the manufacture of rotary endodontic instruments. J Endod. 2012;38(11):1535-40.

5. Abu-Tahun I, Al-Rabab'ah MA, Hammad M, Khraisat A. Technical quality of root canal treatment of posterior teeth after rotary or hand preparation by fifth year undergraduate students, The University of Jordan. Aust Endod J. 2014;40(3):123-30.

6. Peru $M$, Peru $C$, Mannocci $F$, Sherriff $M$, Buchanan LS, Pitt Ford TR. Hand and nickeltitanium root canal instrumentation performed by dental students: a micro-computed tomographic study. Eur J Dent Educ. 2006;10(1):52-9.

7. Martins RC, Seijo MO, Ferreira EF, Paiva SM, Ribeiro Sobrinho AP. Dental students' perceptions about the endodontic treatments performed using NiTi rotary instruments and hand stainless steel files. Braz Dent J. 2012;23(6):729-36.

8. Ungerechts $C$, Bårdsen A, Fristad I. Instrument fracture in root canals - where, why, when and what? A study from a student clinic. Int Endod J. 2014;47(2):183-90.

9. Zarpelon FI, Barp EN, Galafassi D, Melo TAF. Analysis of preparation time, wear, transportation and centering ability of Flexofile and Prodesing $\mathrm{M}$ manual instruments in curved root canals preparation. Rev Odonto Cienc. 2018;33(1):58-63

10. Rolland S, Hobson R, Hanwell S. Clinical competency exercises: some student perceptions. Eur J Dent Educ. 2007; 11(3):184-91.

11.Lanning SK, Wetzel AP, Baines MB, Ellen Byrne B. Evaluation of a revised curriculum: a four-year qualitative study of student perceptions. J Dent Educ.2012;76(10):1323-33. 


\section{CONFLITO DE INTERESSES}

Os autores declaram não haver conflitos de interesse

\section{AUTOR PARA CORRESPONDENCIA}

Alexandre Moreira Fernandes

Centro Universitário Newton Paiva,

30341-262 Belo Horizonte - MG, Brasil

E-mail: alexandre.fernandes@newtonpaiva.br

Submetido em 30/11/2019

Aceito em 22/10/2020 